\title{
Antivaccination movement exploits public's distrust in scientific authority
}

An editorial error led to wrong email address being included in this letter (BMJ 2019;367:16960, doi:10.1136/bmj.16960). The correct email address is: mgolden@ uoguelph.ca. This has been corrected online. 